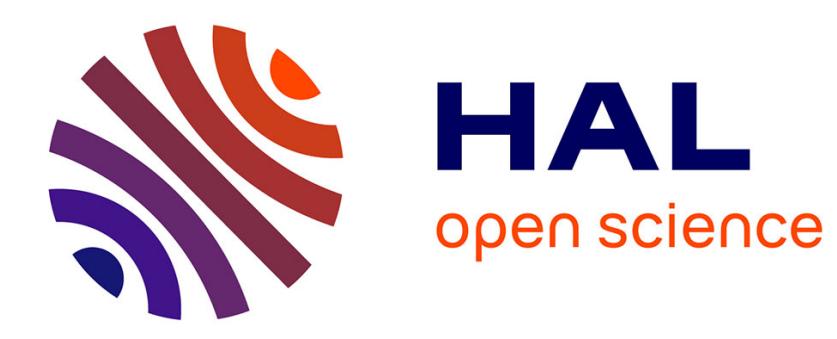

\title{
Les phénomènes de tassement du sol forestier dus à l'exploitation mécanisée du bois
}

Ciceron Rotaru

\section{To cite this version:}

Ciceron Rotaru. Les phénomènes de tassement du sol forestier dus à l'exploitation mécanisée du bois. Revue forestière française, 1985, 37 (5), pp.359-370. 10.4267/2042/21823 . hal-03423734

\section{HAL Id: hal-03423734 \\ https://hal.science/hal-03423734}

Submitted on 10 Nov 2021

HAL is a multi-disciplinary open access archive for the deposit and dissemination of scientific research documents, whether they are published or not. The documents may come from teaching and research institutions in France or abroad, or from public or private research centers.
L'archive ouverte pluridisciplinaire HAL, est destinée au dépôt et à la diffusion de documents scientifiques de niveau recherche, publiés ou non, émanant des établissements d'enseignement et de recherche français ou étrangers, des laboratoires publics ou privés. 


\title{
LES PHÉNOMÈNES DE TASSEMENT DU SOL FORESTIER DUS À L'EXPLOITATION MÉCANISÉE DU BOIS
}

\author{
C. ROTARU
}

Une prise de conscience de la nécessité d'accorder une plus grande attention aux problèmes posés par le tassement du sol forestier résulte des conséquences négatives des dégâts d'exploitation sur l'évolution des forêts.

Depuis 1980, le Centre technique du bois et de l'ameublement a retenu comme un de ses principaux thèmes d'études, les interactions entre l'exploitation forestière et la sylviculture. Le présent article concerne les phénomènes de tassement du sol forestier, leurs causes, et fait des recommandations pour en limiter les effets.

\section{PROBLÈMES POSÉS PAR L'EXPLOITATION FORESTIÈRE}

Les machines d'exploitation forestière travaillent dans des conditions difficiles, parfois en raison des pentes et de la faible portance des sols, mais souvent du fait du grand nombre d'obstacles tels que : fossés, rochers, souches, ainsi que de la végétation importante qui assez fréquemment existe encore au moment de l'exploitation (arbres, arbustes, semis à protéger, etc...). En principe, les machines ne devraient pas provoquer de tassement du sol forestier. Mais, elles doivent aussi vaincre les obstacles évoqués précédemment, d'où deux exigences à concilier. Une machine de type forestier doit avoir de bonnes aptitudes en pentes, en rampes, de bonnes qualités pour le franchissement d'obstacles: débattement, articulation, garde au sol et enfin, une bonne maniabilité, ceci ımpliquant surtout un rayon de braquage réduit. De plus, tout engin doit permettre d'obtenir une bonne productivité.

II faut constater aussi une évolution vers une augmentation de la puissance des tracteurs forestiers. Par exemple, en matière de tracteur-débusqueur, la puissance des matériels les plus vendus en France actuellement est de 100 à $120 \mathrm{ch}$ au lieu de $80 \mathrm{ch}$, il y a une dizaine d'années. De même, on constate souvent une augmentation du poids des machines d'exploitation du bois, comme par exemple des débusqueurs ou porteurs qui dépassent quelquefois 14 tonnes à vide. Mais il ne faut pas croire que le tassement du sol forestier est seulement l'effet direct résultant de la pénétration des engins d'exploitation forestière sur la coupe. Le sol a été auparavant plus ou moins tassé par telle ou telle intervention culturale ou par le piétinement des hommes ou des animaux. Au moment de l'exploitation, les machines qui interviennent peuvent maintenir ou aggraver une situation bonne ou mauvaise. Aussi, la responsabilité de l'exploitant doit-elle être mesurée avec beaucoup d'attention. 


\section{ROTARU}

L'argument productivité a compté aussi beaucoup dans le choix des grosses machines en forêt. C'est pour cela, et aussi pour une meilleure valorisation de l'arbre abattu, que se sont développées des méthodes d'exploitation comme celle de l'arbre entier ou celle en grandes longueurs. C'est ainsi, par exemple, qu'on voit souvent dans les Vosges et le Jura des grumes de 24 et $25 \mathrm{~m}$, débusquées en bord de route et tronçonnées ensuite à $18 \mathrm{~m}$. Le désir normal d'une bonne productivité pour l'exploitant, s'ajoute à une recherche analogue du transporteur et du scieur, qui pensent mieux tirer profit des grandes longueurs.

La mécanisation en forêt est un facteur de progrès social incontestable mais il faut être bien conscient de ses limites et de ses conséquences sur l'avenir des peuplements. Nous nous limiterons ici aux incidences sur les sols.

\section{PERTURBATION DU CYCLE BIOLOGIQUE EN FORÊT}

Le tassement du sol forestier peut constituer un des facteurs importants de la perturbation du cycle biologique de la forêt. D'autres facteurs peuvent s'ajouter à cette perturbation ou agir de manière indépendante sans tassement du sol comme, par exemple, l'accumulation des feuilles mortes en couches épaisses et mal décomposées dans certaines futaies d'essences à couvert épais (Hêtre, Sapin, Epicéa), un sol trop humide, trop acide ou trop pauvre.

Le tassement du sol forestier peut influencer de manière négative la vie des arbres par: le ralentissement à différents degrés du cycle biologique, une réduction de l'activité racinaire, la mauvaise aération, le ralentissement d'activité métabolique des radicelles et des micro-organismes associés, les difficultés de germination des graines qui tombent sur la surface du sol, etc...

Le ralentissement du cycle biologique est une conséquence du tassement qui se manifeste d'abord dans les horizons des couches supérieures, dites holorganiques $(F, H)$. La réduction de la porosité, la mauvaise circulation de l'eau et de l'air, empêchent la vie d'organismes transformateurs (microflore et microfaune) et conduisent à des blocages dans la chaîne trophique. La liberation des éléments nutritifs à partir de cette zone (minéralisation), vers les horizons hémorganiques (A1) ou minéraux ( $A 2,(B), B \ldots$ ) où se trouvent concentrées les racines alimentant l'arbre, est réduite. Cette réduction dans la libération des éléments nutritifs est très variable; elle est fonction du degré de tassement du sol, et de l'association éventuelle d'autres tacteurs de perturbation du cycle tels que ceux qui sont évoqués précédemment.

Les conséquences de cette réduction se manifestent dans la croissance des arbres (Fries, 1975). En principe, si la minéralisation de la litière est lente, l'horizon Ao augmente d'épaisseur chaque année, et un humus brut ou "mor", peu favorable à la croissance des arbres, se constitue. On a de fortes présomptions de croire que ces mêmes phénomènes négatifs peuvent diminuer les qualités technologiques $d u$ bois en provenance des zones perturbées par le tassement excessif $d u$ sol forestier, mais des recherches à long terme seront nécessaires pour confirmer cette hypothèse qu'il faut considérer avec beaucoup de précaution.

Un degré extrême de perturbation du cycle biologique est atteint lorsque le tassement excessif qui arrive à s'exercer sur une grande surface a des répercussions, non seulement dans les couches supérieures du sol, mais aussi en profondeur, dans les horizons hémorganiques et minéraux. Ce phénomène se traduit par un affaiblissement de la défense naturelle des arbres face aux attaques de différents agents pathogènes et, en définitive, il peut entraîner leur dépérissement. Dans la plupart des cas, cette évolution négative est le résultat de plusieurs facteurs perturbateurs du cycle biologique, parmi lesquels le tassement excessif est un des plus importants. 


\section{LES PHÉNOMÈNES DE TASSEMENT DU SOL FORESTIER}

Avant de commenter les phénomènes de tassement du sol forestier provoqués par les engins d'exploitation forestière, il faut rappeler que le comportement du sol soumis au tassement dépend surtout :

- de sa texture (proportions d'éléments fins ou microfins, argiles, limons, sables),

- de sa structure, et en particulier de la stabilité de celle-ci, c'est-à-dire de la façon dont sont assemblées les particules élémentaires qui le constituent,

- de sa teneur en matière organique,

- de son humidité.

Sans exposer chacune de ces caractéristiques propres du sol, en les supposant connues, il faut examiner les phénomènes spécifiques qui interviennent toujours lorsqu'il s'agit du problème de tassement du sol forestier:

- le scalpage (effet de fraisage et de laminage à la surface du sol),

- l'orniérage (effet de creusement à la surface et en zone moyenne du sol),

- le martelage (effet de tassement extensif à la surface du sol),

- le compactage (effet de tassement excessif du sol).

\section{Le scalpage du sol forestier}

Le phénomène de scalpage du sol forestier (photo 1) est provoqué par le glissement des roues motrices qui tournent sans avancer, ou en avançant très peu par suite d'un manque d'adhérence ou de portance, ou du fait du trainage ou semi-trainage sur le sol de grumes mal préparées (sans parage). Le résultat de ces actions n'est pas seulement l'arrachage et la destruction de la litière, mais aussi d'une couche supérieure du sol, qui est également soumise à un tassement structurel. A cette occasion, une partie des racines qui se trouvent à la surface du sol sont détruites. Dans le domaine agricole, on emploie pour ce phénomène le terme de cisaillement. Dans le domaine forestier, le terme scalpage a été employé à cause de la litière, laquelle n'existe pas sur les terres agricoles.

Des études sur l'adhérence et la portance des engins sur le sol forestier ont mis en évidence le fait que la forme et le profil et,

Photo 1 : Scalpage du sol forestier.

Photo C. ROTARU

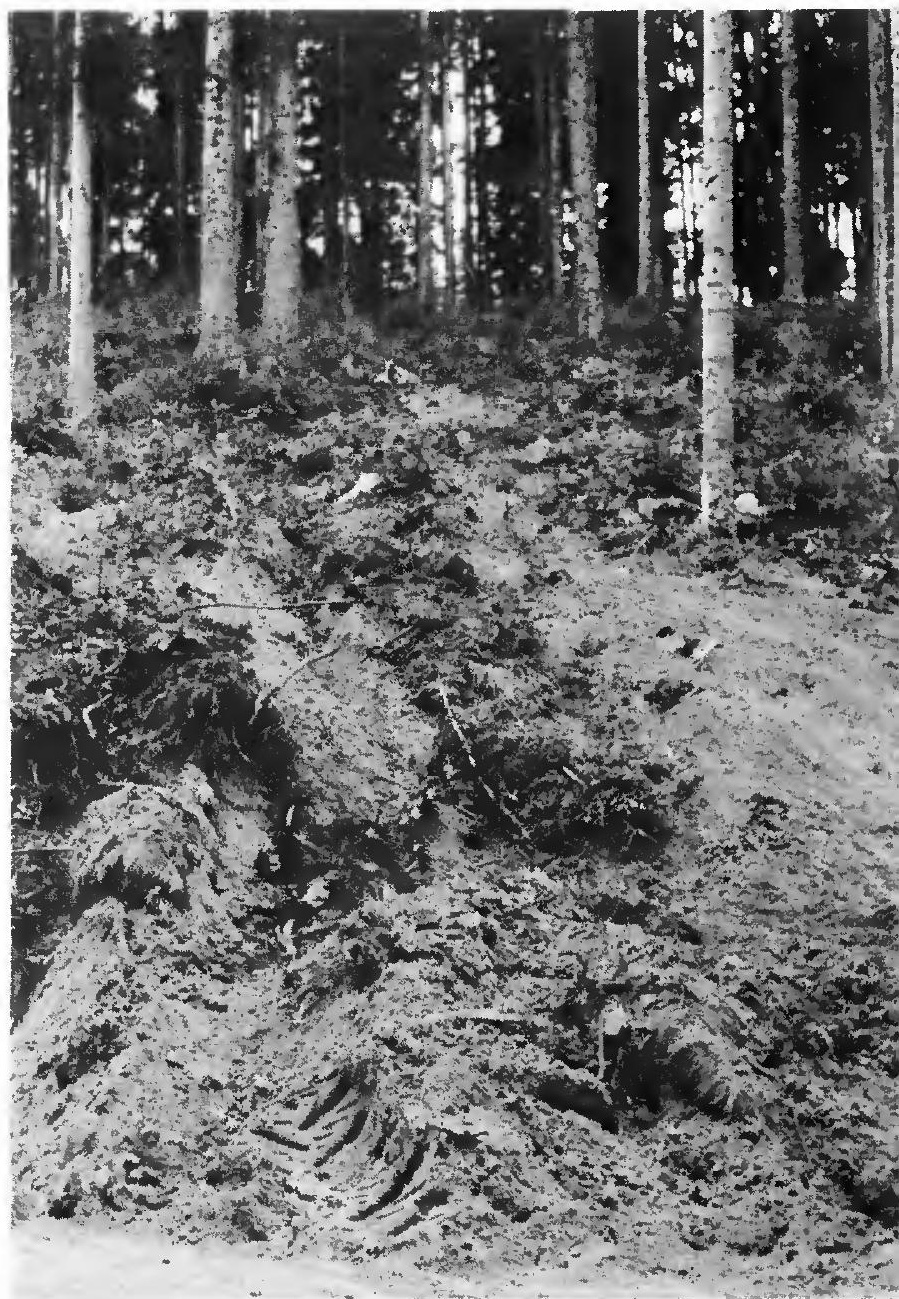




\section{ROTARU}

corrélativement, la surface de contact des pneus, en dehors du type et de l'état du sol, sont aussi des facteurs de très grande importance. De ce point de vue, il faut signaler les recherches de $P$. Abeels sur des pneumatiques adaptés aux travaux forestiers. En principe, on peut retenir, pour l'emploi des pneus ${ }^{(1)}$ dans le domaine forestier, les recommandations suivantes:

- l'effort de traction augmente avec la surface de contact, sauf pour des sols de cohésion très faible,

- le coefficient d'adhérence augmente si la pression unitaire exercée sur le sol diminue.

Les nervures des pneumatiques présentent peu d'intérêt pour les sols peu cohérents (sableux par exemple) et elles sont mal adaptées pour les sols très durs (contact discontinu entre roue et sols). En revanche, pour la majorité des sols forestiers de cohésion moyenne, le rôle des nervures des pneumatiques est important parce qu'elles permettent d'atteindre une couche sous-jacente, en général plus ferme que la litière superficielle.

\section{L'orniérage du sol forestier}

Un autre aspect du tassement, que l'on peut trouver en forêt, est représenté par les ornières laissées par le passage des roues. Une ornière comprend un creux (ornière proprement dite), et souvent un ou deux bourrelets latéraux.

Au centre de l'ornière, on observe des zones de feuilletage fin et feuilletage grossier, résultant de la modification structurale du sol, accompagnées quelquefois par une coalescence des grumeaux (agglomérés de particules) et des mottes (agglomérés de grumeaux).

On peut distinguer trois grands types d'ornières:

- Ornière avec bourrelets latéraux, sans tassement (type 1)

Pour ce type d'ornière, le volume du creux est presque égal au volume des bourrelets et en principe elle ne dépasse pas $10 \mathrm{~cm}$ de profondeur;

- Ornière avec bourrelets et tassement (type 2)

Dans ce cas, le volume du creux est plus grand que le volume des bourrelets et en principe, sa profondeur est de 10 à $30 \mathrm{~cm}$;

- Ornière sans bourrelet latéral mais avec tassement (type 3)

Ce type d'ornière présente une situation plus complexe du point de vue tassement, les particules du sol subissant un fort déplacement vertical.

Ici, la déformation est faible mais le tassement est important. Un signe évident de reconnaissance est la persistance de l'eau stagnante dans le creux de l'ornière pendant des semaines ou des mois (photo 2).

La sensibilité du sol au tassement est liée non seulement à sa texture et à sa structure, mais aussi à l'humidité qui contribue à la plasticité du matériau. De ce point de vue, on peut constater deux situations :

- la pression localisée est inférieure à la pression limite caractérisant la portance du sol. Celui-ci reste dans un état de pseudo-élasticité ; la trace du pneu provoque une faible déformation ;

(1) Pour plus de précisions sur l'utilisation des pneus sur terres forestieres, voir les articles du Professeur P. Abeels 


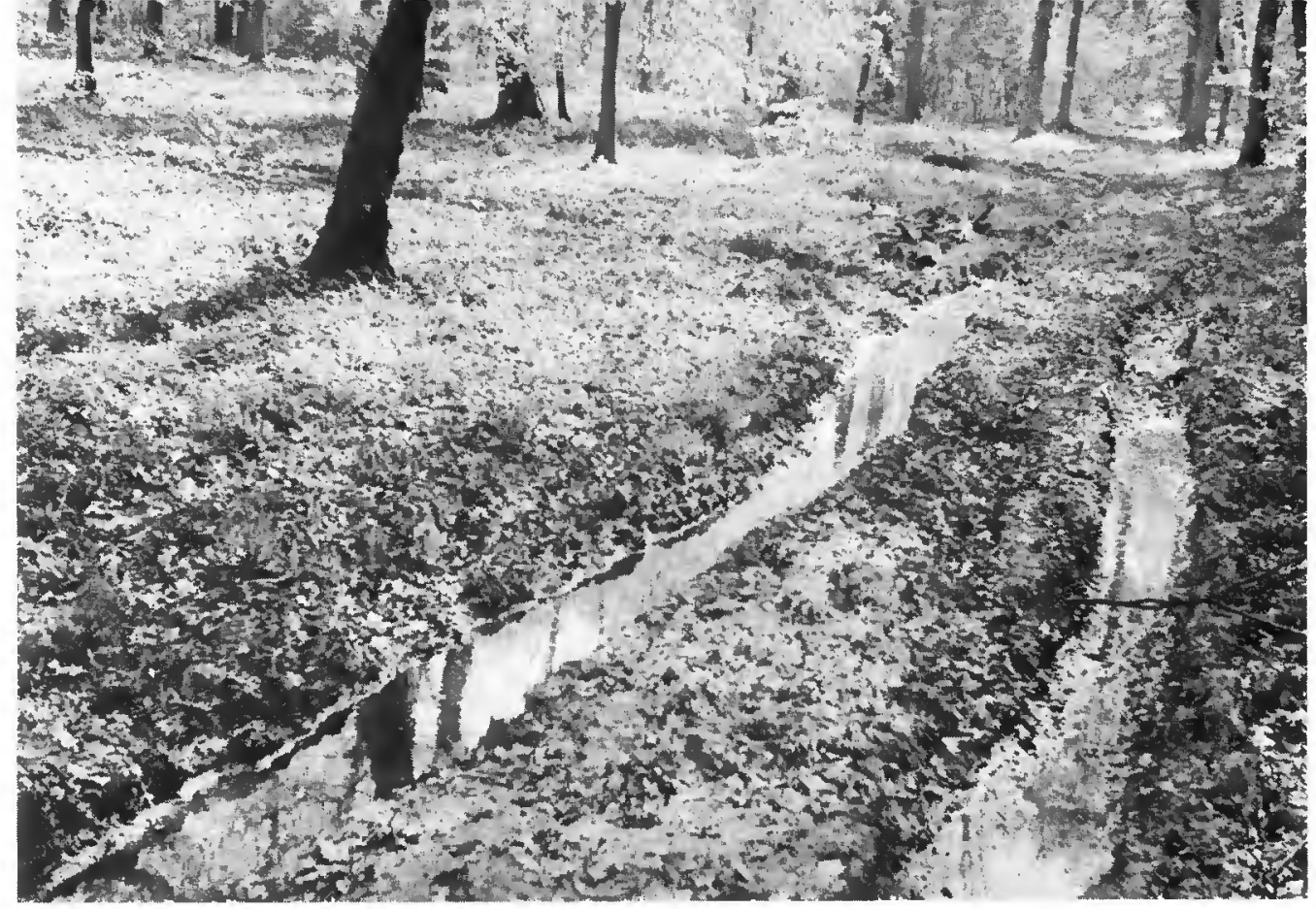

Photo 2 : La persistance de l'eau dans le creux des ornières est un signe de tassement excessif (ornière de type 3).

Photo C. ROTARU

- la pression localisée est supérieure à la pression limite, le sol entre en état de plasticité et la trace observèe sur le terrain est une véritable ornière de profondeur plus ou moins importante (photo 2).

On peut donc souligner le fait que la profondeur d'une ornière dépend, d'une part, de la structure du sol et de son humidité et, d'autre part, du poids de l'engin, de la surface de contact des pneus ou chenilles avec le sol et du nombre de passages effectués (du trafic). Le tassement du sol est cumulatif et l'effet maximal ne s'obtient qu'après un certain nombre de passages.

Sans travaux d'amélioration, la densité brute initiale ne revient (Dikerson, 1976), dans les zones tassées par orniérage ou trainage, qu'après 8 à 12 ans (figure 1).

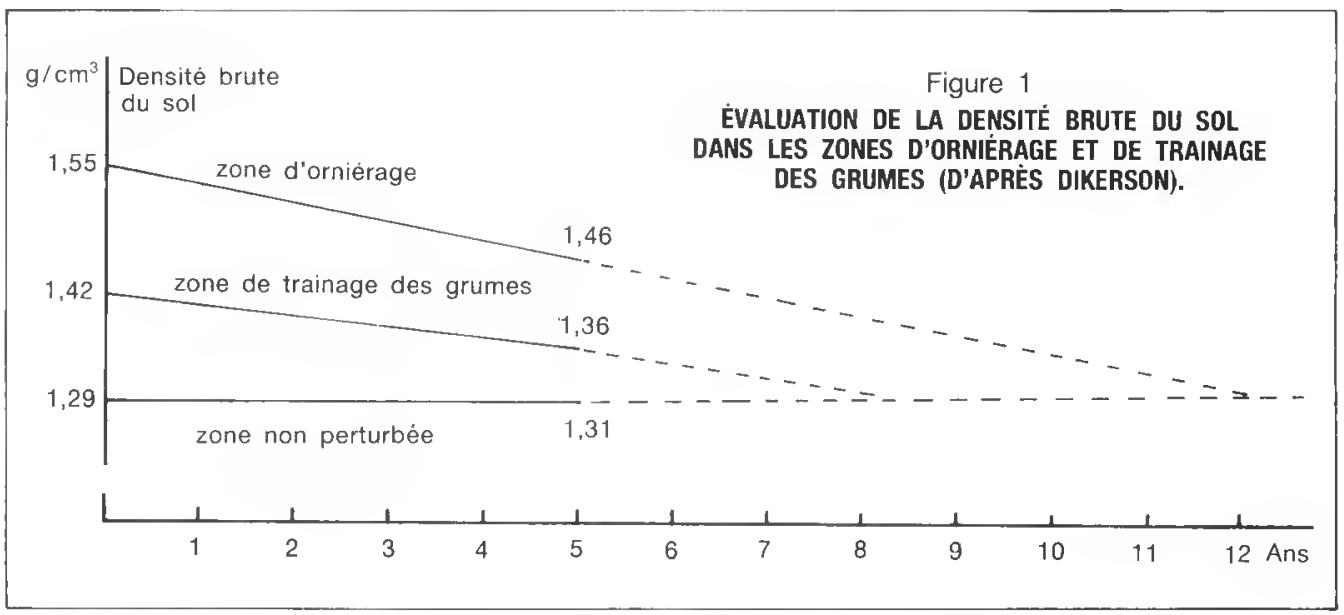




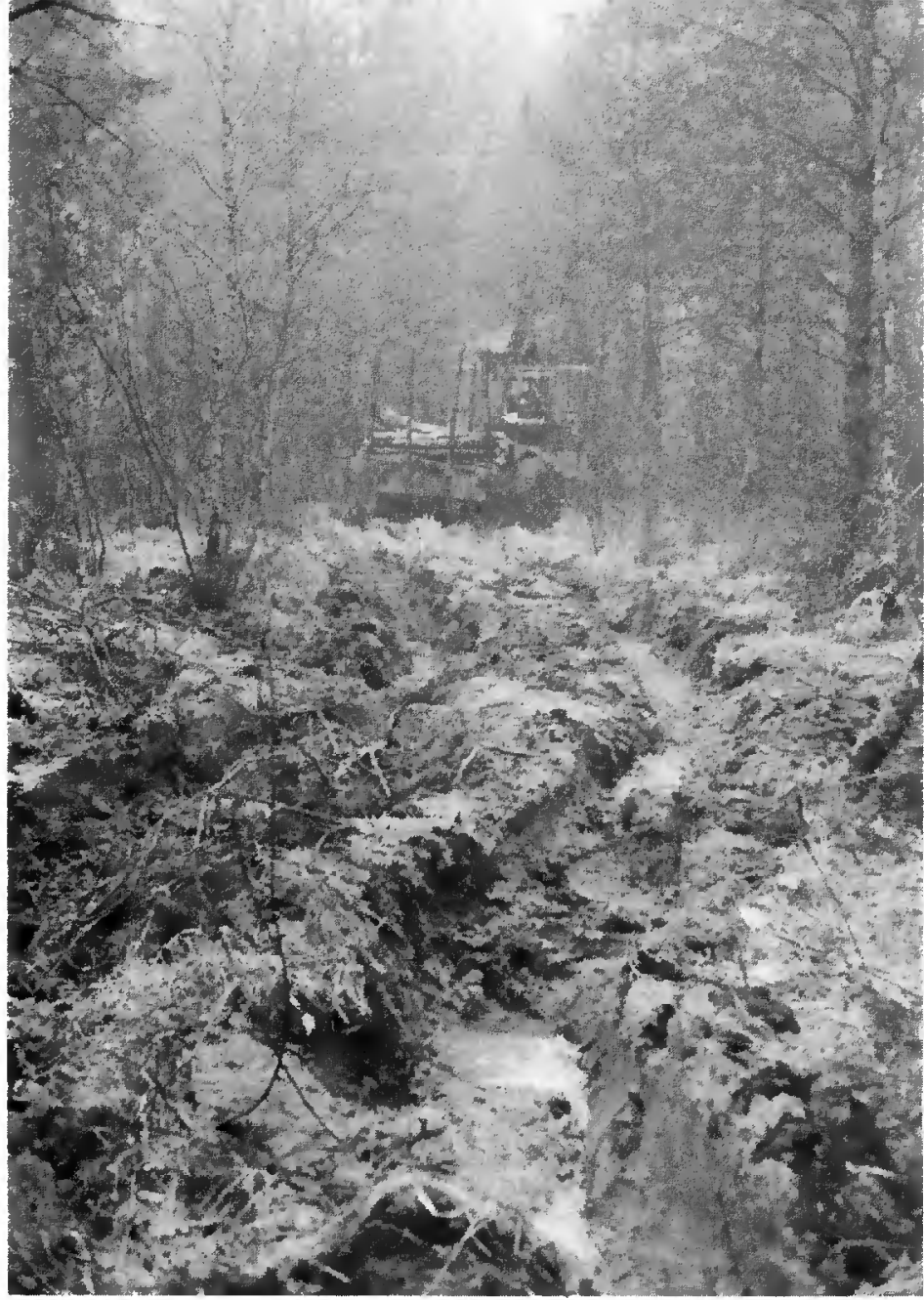

Photo 3: Ornièrage accompagné du scalpage du sol sur un couloir de dèbardage dans une coupe d'éclaircie et qui a eu comme effet direct la destruction d'un grand nombre de racines des arbres avoisinants.

Photo C. ROTARU

II n'est pas rare de constater que l'orniérage est accompagnè de scalpage, comme on peut le voir d'après la photo 3 , et a comme résultat la destruction d'un grand nombre de racines des arbres avoisinants. Le plus souvent, ces phénomènes sont provoqués par les tracteurs agricoles à deux roues motrices seulement, en principe mal adaptėes pour le travail en forêt et sur sol mouillé.

\section{Le martelage du sol forestier}

Quelquefois, à la surface d'une parcelle, on peut constater le tassement du sol non seulement sur le trajet des pistes, mais partout oú les engins avec des charges importantes sont passés de façon répétée (action de rouleau compresseur), spécialement en périodes sèches, et n'ont pas laissé des ornières importantes. On peut donc avoir un tassement de sol de surface "extensif ", qu'on appelle "martelage du sol "(tassement dynamique), signale surtout sur des sols qui ont une couche dure proche de la surface et où les résonances des pressions n'ont pu être bien résorbées. On considère que cette forme de tassement peut se produire même si les pressions exercées ne sont pas excessivement grandes. Par différence avec le compactage, cette forme de tassement est réversible, et des travaux d'amélioration de sol (coûteux) seront nécessaires pour ne pas affecter la croissance d'une autre génération d'arbres (photo 4).

\section{Le compactage du sol forestier}

Si plusieurs passages se produisent aux mêmes endroits, il y a répétition des mises en charge, avec écrouissage et déformation cumulés, pouvant atteindre la zone de déformation plastique, et laisser une ornière profonde avec tassement important qui modifie de manière très importante la porosité du sol; ce qui conduit à une prise en masse totale de celui-ci : c'est le compactage (photo 5) qui est un tassement statique (difficilement réversible). 


\section{ROTARU}

Le phénomène de compactage est d'une grande complexité. En principe, il représente l'effet maximal du tassement (cumulatif), et on le trouve dans une zone située à l'intérieur du sol. Lorsqu'on parle de pression localisée exercèe par un pneu, on parle en réalité d'une pression moyenne. A cause des nervures (crampons), et en fonction d'autres données de fabrication, un pneu présenté comme exerçant $1,8 \mathrm{~kg} / \mathrm{cm}^{2}$ peut exercer des pressions localisées pouvant atteindre $12 \mathrm{~kg} / \mathrm{cm}^{2}$, voire davantage sur terrain sec.

Les pressions localisées dans les zones d'orniérage et de trainage se diffusent en profondeur (figure 2) ou, par effet cumulatif suite au passage répété des engins lourds, peuvent provoquer le compactage (les zones d'argile sont les plus sensibles). Ce phénomène de diffusion des pressions s'appelle aussi résonance des pressions.

\section{Figure 2 : LA DIFFUSION DES PRESSIONS LOCALISĖES À LA SURFACE DU SOL SUITE AU PASSAGE RÉPÉtE DES ENGINS LOURDS PEUT PROVOQUER EN PROFONDEUR LE PHENOMENE DE COMPACTAGE.}

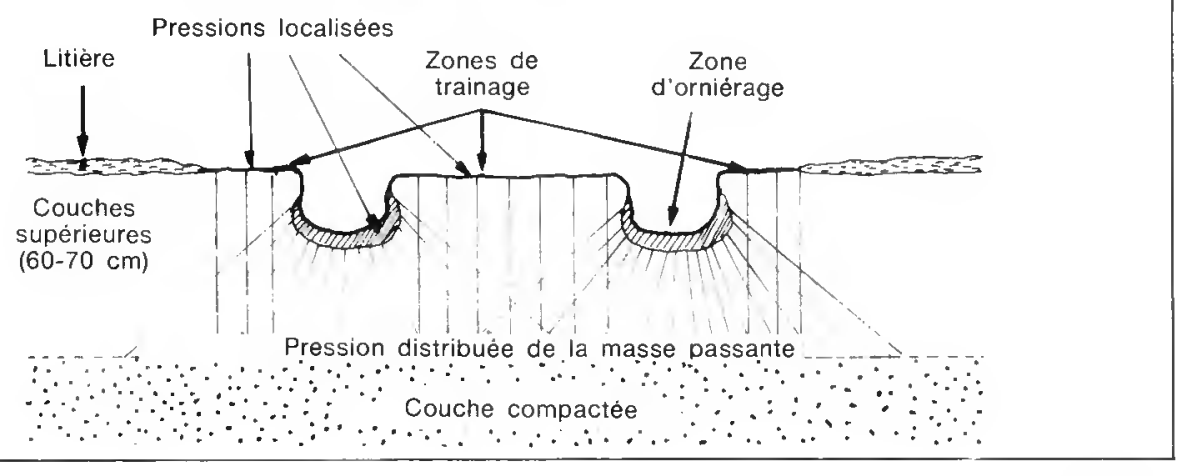

Le compactage qui affecte l'assemblage des particules élémentaires est difficilement réversible, et il est dangereux pour le bon fonctionnement du système racinaire, comme on peut le constater d'après la figure 3 , réalisée dans le cadre d'une étude sur l'effet de ce type de tassement sur le système racinaire, effectuée aux Etats-Unis d'Amérique par les laboratoires de recherches Caterpillar.

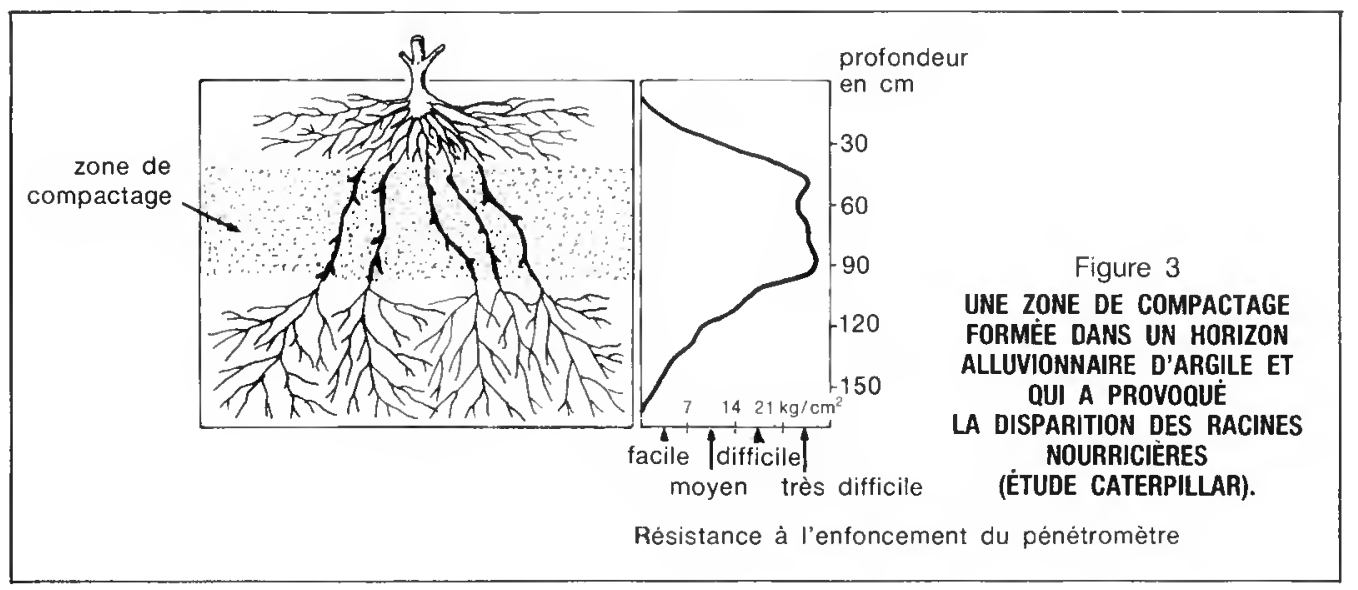


Du fait du compactage, l'imperméabilisation d'une couche du sol en profondeur provoque un excès d'humidité dans les couches supérieures et à la surface du sol. Cette situation a aussi des conséquences négatives sur la vie du sol et des arbres (asphyxie des sols). Comme signes extérieurs de compactage, on peut observer l'eau stagnante dans certains endroits plats ou concaves, et un jaunissement des feuilles dans les zones affectées.

A l'intérieur du sol, on observe une prolifération des racines juste au-dessus de la zone compactée, et les racines poussent même horizontalement si le compactage est élevé.

\section{RECOMMANDATIONS}

Pour éviter, ou réduire, les différents phénomènes de tassement du sol forestier, il faut retenir les principes suivants:

- Si l'engin doit circuler sans restriction sur le parterre de la coupe (coupe à blanc), il est souhaitable de le doter de pneus ou de chenilles qui n'exercent en charge qu'une faible pression au sol (maximum de $0,7 \mathrm{~kg} / \mathrm{cm}^{2}$ ) (photo 6). En absence de tels équipements, on peut craindre des dommages importants dus au tassement sur des sols mouillés.

- Si les sols sont gorgés d'eau, c'est-à-dire ont une teneur très supérieure à la capacité au champ, il faut éviter de débarder, ou prendre des dispositions spéciales pour limiter le passage des engins, même les mieux adaptés, dans la parcelle.

- La circulation des engins de débardage dotés de pneus à haute pression de gonflage et qui exercent une pression èlevée sur le sol doit se faire seulement sur les voies forestières pour limiter les dégâts. Le bois sera ramené à l'engin par treuillage, ou à l'aide de grues à longue flèche. Si la piste de débardage a èté endommagée par des ornières, l'exploitant aura l'obligation de la réparer.

Photo 6: Les porteurs à 8 roues motrices dotées de pneus larges exercent une faible pression sur le sol forestier.

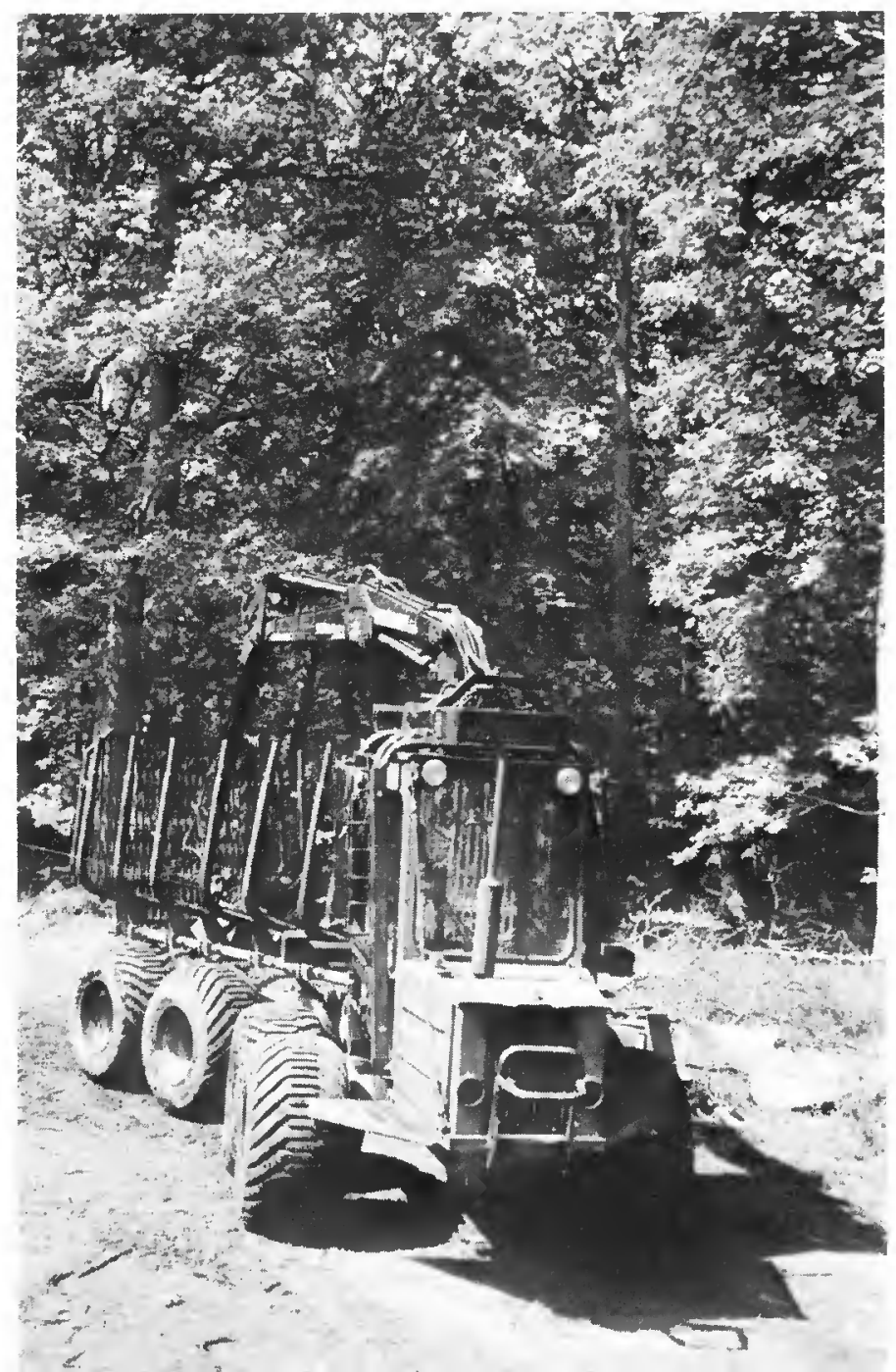




\section{ROTARU}

- Dans les forêts situées sur des terrains à faible pente ou en régions de plaine, il y a toujours des trajets très fréquentés par les engins de débardage sur les axes qui débouchent au dépôt primaire. Il est nécessaire pour éviter l'orniérage profond et la casse des engins, du fait de leurs passages renouvelés dans ces zones, d'empierrer les trajets en question en les considérant comme routes secondaires.

- Pour éviter ou réduire les dommages que peut provoquer le débardage sur sol mouillé, il est recommandé de disposer les rémanents sur les pistes ou les cloisonnements et de ne pas quitter ces voies de circulation.

- La définition d'un réseau de routes forestières réalisées dans des terrains en pente de plus de $30 \%$ doit aussi tenir compte des possibilités de débardage des bois par voie aérienne, moyen important pour éviter les différents phénomènes du tassement du sol. Dans ce sens, il faut souligner l'importance des installations mobiles à câbles pour éviter un réseau trop dense de routes, surtout en région montagneuse.

- Il faut créer des places de dépôt même si cela n'a pas été prévu par l'aménagement. En principe, un dépôt primaire doit se trouver en bordure de la coupe et en même temps, en bordure d'une route forestière. Ainsi, sera évité le débardage à longue distance qui a une incidence négative sur le sol et sur les coûts d'exploitation.

- L'organisation préalable correcte des travaux d'exploitation permet d'assurer une bonne corrélation entre les travaux de bûcheronnage et de débardage, et de disposer d'une voirie de vidange correcte, tracée d'avance (la constitution des piles de bois d'industrie et de bois de teu doit se faire près de ces voies).

- Il est indispensable d'instruire et informer les conducteurs d'engins sur les problèmes de tassement du sol.

- $\mathrm{Si}$, en raison de travaux de débardage renouvelés, du piétinement ou d'autres causes, le sol est tassé et la litière ne peut pas se décomposer, il faut procéder à des travaux de crochetage dont le rôle est d'améliorer la qualité des horizons de surface. Le crochetage mécanique est toujours préférable au crochetage manuel.

- Les travaux de sous-solage peuvent constituer un procédé efficace pour améliorer les sols tassés, facilitant en effet la pénétration de l'eau en profondeur sans que cela ramène en surface les horizons profonds à mauvaise structure. De tels procédés doivent être accompagnés par des mesures sylvicoles à longue échéance telles que: la création d'un sous-étage de feuillus améliorants ou de peuplements mélangés, la bonne conduite d'éclaircies, etc...

\section{CONCLUSIONS}

Le problème du tassement du sol forestier préoccupe de plus en plus les spécialistes du monde entier. II faut remarquer que les plus vives réactions commencent à se produire dans les grands pays forestiers où, souvent, la mécanisation a été poussée à l'extrême limite (gigantisme des engins de débardage, voire d'autres matériels).

En France, on ne peut pas dire que des excès importants aient été commis, et cela grâce à une action conjointe des gestionnaires et des exploitants professionnels. Pour préserver l'avenir des forêts, surtout dans le cas d'une extension de la mécanisation, il faut bien connaître les problèmes posés par le tassement du sol, et en tirer des conclusions. Comme on l'a vu, il n'est pas possible de proposer une solution unique évitant le tassement du sol forestier. II faut avoir toujours en vue un ensemble de mesures prenant en compte le matériel, l'organisation des travaux, les caractéristiques des forêts ainsi que les qualités et les connaissances du conducteur. Beaucoup de solutions pratiques, liées aux caractéristiques de chaque coupe, peuvent 
venir de la part du conducteur d'engin, s'il a des connaissances dans ce domaine, et s'il arrive à comprendre que le sol de la forêt est vivant et qu'il ne faut pas le traiter comme un objet inerte capable de tout supporter.

Il est, de fait, qu'on ne peut pas facilement exploiter sans risquer de provoquer certains préjudices à la forêt (blessures aux arbres ou tassement du sol spécialement). Eviter les dégâts d'exploitation en interdisant la circulation des engins sur la coupe n'est guère envisageable. Un autre extrême serait d'admettre en forêt, sans restriction, les engins dont les caractéristiques ne sont pas favorables et de les laisser alors provoquer de grands dégâts. Réduire au maximum possible les préjudices et avoir, en même temps, une productivité convenable est l'apanage d'une science complète de l'exploitation forestière. II arrive qu'on acquiert seulement des connaissances dans le domaine des machines d'exploitation forestière et leurs productivités, ou seulement des connaissances dans le domaine des sols ou de la sylviculture. L'extension de la mécanisation en forêt rend indispensable un enseignement mixte, machine et forêt, si l'on veut pratiquer une foresterie moderne et avec un minimum de risques.

Jusqu'à maintenant, on a constaté surtout une tendance à lier les problèmes de tassement du sol forestier et les dommages causés à la forêt aux caractéristiques des machines et, éventuellement, du fait du conducteur. On oublie quelquefois que les hommes qui travaillent avec les nouveaux moyens doivent être bien formés. On sait aussi que la meilleure machine peut donner les pires résultats, quant à sa productivité et aux dégâts causés en forêt, si elle n'est pas utilisée à bon escient, et si elle n'a pas un conducteur éduqué suivant les principes évoqués précédemment.

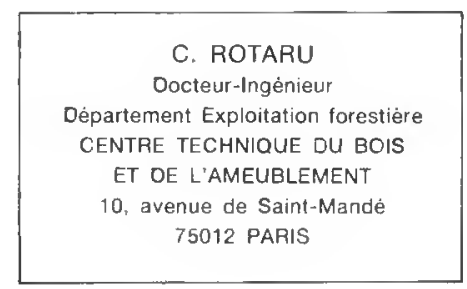

\section{BIBLIOGRAPHIE}

Anonyme. - How much will you lose to soil compaction this year? Publication Caterpillar A.E.D.A.-5 142. ABEELS (P.). - Air cushion vehicles and soil erosion. - Journal of Terramechanics, $n^{\circ} 4,1976$.

ABEELS (P.). - La recherche en matière de locomotion hors-route sur terres agricoles et forestières. Louvain: Université catholique, 1979. - ètude.

ABEELS (P.). - Tyre deflection and contact studies. - Journal of Terramechanics, $n^{\circ} 3,1976$.

BILLOT (J.F.). - La compaction des sols, un problème immèdiat. - France Agricole, août 1981.

BODET (J.M.). - Techniques et matériels pour éviter le tassement du sol (agricole). — Le Producteur agricole français, $\mathrm{n}^{\circ} 2,1981$.

BONNEAU (M.). - Le cycle biologique en forêt. Cours de pédologie forestière. - Nancy : Ecole nationale du Gènie rural, des Eaux et des Forêts, janvier 1980.

DALLEINNE (E.). - Compaction du sol sous differents pneus. - Bulletin C.N.E.E.M.A., n $237,1977$.

DICHERSON (B.). - Soil compaction after treelength skidding in northern Mississipi. Soil Science, Society of America Journal, vol. $40, n^{\circ} 6,1976$.

ERIKSSON (J.). et al. - The effect soil compaction on soil structure an crop yields. Swedish Institute of Agricultural Enginering, Bulletin 354, 1974.

ERIKSSON (L.). - Strip roads and damages caused by machines when thinning stands. - Garpenberg: Sveriges Lantbruksuniversitet, 1981. - rapport 137. 


\section{ROTARU}

FRIES (J.). - Okologische Aspekte der mechanisierten Durchforstung. - Der Forst und Holzwirt, $\mathrm{n}^{\circ} 17,1975$. GIBSON (-), BILLER (-). - Slide slope stability of logging tractors and forwarders. - Transaction of the A.S.A.E., mars-avril 1974.

KERSCHENMAYER (J.). - La compaction des sols. - Cultivar, juin 1981.

KOUASSI (B.). - Phénomènes de tassement du sol sous l'action des engins agricoles. - Bulletin C.N.E.E.M.A., $n^{\circ} 237,1977$.

NIPKOW (F.). - Holzrücken auf schlecht tragfähigen Waidböden: Raupe oder Breitreifen? Allegemeine ForstZeitschrift, $n^{\circ} 4,1983$, p. 947-997.

ROTARU (C.). - Technologia exploatarii lemnului (la technologie de l'exploitation du bois). - Bucarest: Ed. C.D.P.T., 1974.

ROTARU (C.). - Tassemenf du sol forestier et récolte mécanisée. - Paris : Centre technique du Bois/C.E.F.S., $n^{\circ} 1,1983$.

ROTARU (C.). - Comment limiter le tassement du sol en respectant la productivité ? - Le bois national, $\mathrm{n}^{\circ}$ 22, 16 juin 1984, pp. 29-30.

ROTARU (C.). - interactions entre les méthodes d'exploitation et la sylviculture. - Paris: Centre technique du Bois, C.E.F.S., $n^{\circ} 2,1984$.

TOUTAIN (F.). - Les humus forestiers. - Revue forestière française, vol. XXXIII, $n^{\circ} 6,1981, p p .449-479$.

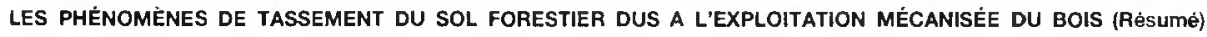

L'article fait une description et une analyse sur les causes de phénomènes du tassement du sol forestier suite á l'exploitation forestière. L'auteur classifie ces phenomènes ainsi:

- le scalpage (effet de fraisage et de laminage à la surface du sol, qui peut avoir comme effet direct la destruction d'une partie des racines qui se trouvent dans les couches superieures),

- l'orniérage (effet de creusement á la surface et en zone moyenne du sol),

- le martelage (effet de tassement extensif à la surface du sol),

- le compactage (effet de tassement excessif du sol).

Sont expliquées les conséquences sur la forêt et sont données les solutions pour réduire les risques du tassement du sol forestier.

\section{COMPRESSION PHENOMENA IN FOREST SOILS DUE TO MECHANIZED LOGGING (SUmmary)}

The article describes and analyses the causes of compression phenomena in forest soils that result from logging. The author classities these phenoma as follows:

- scalping : the effect of abrasion and lamination at the surface of the soil, which may have the direct results of destroying a part of the roots that are in the top layers.

- rut formation: furrowing at the surface and medium depths of the soil,

- beating : the effect of extensive compression at the soil surface,

- compaction: the effect of excessive compression at the soil surface soils.

The consequences for the forest are explained and methods are given for reducing the incidence of compression in torest

DIE EASCHEINUNGEN DER SENKUNG DES WALDBODENS INFOLGE DER MECHANISIERTEN HOLZBRINGUNG (ZuSAmmENFASsung)

Der Beitrag beschreibt und untersucht die Ursachen des Auftretens von Senkungserscheinungen des Waldbodens infolge der mechanisierten Holzbringung. Der Verfasser teilt die Erscheinungen folgendermassen ein :

- die Skalpierung (Fräs- und Walzeindruck an der Waldbodenoberfläche dessen direkte Auswirkung die Zerstörung eines Teils des Wurzelwerks in den oberen Schichten sein kann),

- die Furchung (Eingrabungen der oberen und mittleren Bodenzone),

- die Hämmerung (ausgedehnte Verdichtung an der Bodenoberfläche),

- die Pressung (übermässige Bodenverdichtung).

Die Folgen für den Wald werden erläutert und Lösungen zur Reduzierung der Risiken für den Waldboden angegeben.

LOS FENÓMENOS DE APISONAMIENTO dEL SUELO FORESTAL DEBIDOS A LA EXPLOTACIÓN MECANIZADA DEL BOSOUE (Resumen)

El articulo realiza una descripción y un análisis de las cáusas de los fenómenos del apișonamiento del suelo forestal como consecuencia de la explotación del bosque. El autor clasifica dichos fenómenos asi :

- El escalpado (efecto de fresado y de laminado en la superficie del suelo, que puede tener como efecto directo la destrucción de una parte de las raices que se encuentran en las capas superiores),

- El carrileo (efecto de excavación en la superficie y en zona media del suelo)

- El martilleo (efecto de apisonamiento extensivo en la superficie del suelo),

- El compactado (efecto de apisonamiento excesivo del suelo).

Son explicadas las consecuencias sobre el bosque y son dadas las soluciones para reducir los riesgos de apisonamiento del suelo forestal. 